\title{
Rare retroperitoneal conditions that mimic uterine myoma
}

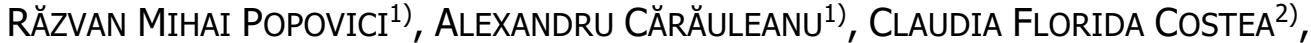

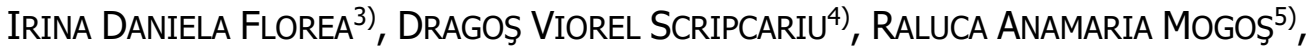

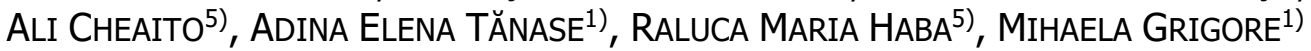 \\ 1)Department of Obstetrics and Gynecology, Faculty of Medicine, Grigore T. Popa University of Medicine and Pharmacy, Iaşi, \\ Romania \\ 2) Department of Ophthalmology, Faculty of Medicine, Grigore T. Popa University of Medicine and Pharmacy, Iaşi, Romania \\ 3) Department of Immunology, Faculty of Medicine, Grigore T. Popa University of Medicine and Pharmacy, Iaşi, Romania \\ 4) Department of General Surgery, Faculty of Medicine, Grigore T. Popa University of Medicine and Pharmacy, Iaşi, Romania \\ ${ }^{5)}$ Cuza Vodă Hospital of Obstetrics and Gynecology, Iaşi, Romania
}

\begin{abstract}
The most frequent tumoral condition of the uterus is represented by uterine myoma. The diagnosis, in most cases, is established by clinical examination and ultrasound scan. Nevertheless, there are rare cases, in which the surgical findings reveal a retroperitoneal tumor instead of a uterine myoma. These could be represented by schwannomas or Castleman disease. The schwannomas are rarely malignant and arise from the Schwann cells of nerve fibers. These tumors are frequently found at the level of the head, neck and mediastinum and rarely in the pelvis. Generally, schwannomas localized at retroperitoneal level are asymptomatic and with a very slow growth rate. The treatment consists in complete surgical resection. The recurrence rate is low and, generally, the prognosis is good. The Castleman disease is considered a rare entity, but it should be always taken into consideration when it comes to a differential diagnosis in a young patient who presents a retroperitoneal mass at imagery exams. The condition affects the lymphatic system and is characterized by a hyperplasia of the lymph nodes, sometimes associated with herpes virus infection. The clinical picture is often non-specific; the pain may be the only symptom. The imaging methods are not always conclusive for the final positive diagnosis and the histopathological examination is always necessary. Pelvic Castleman disease can be misdiagnosed as myoma or an adnexal tumor. In this article, we review the present knowledge regarding the pathogenesis, pathology and management of these rare retroperitoneal tumors. Both conditions, when located in pelvis must be taken into consideration in the differential diagnosis of uterine myomas, especially in the pedunculated form.
\end{abstract}

Keywords: uterine myoma, schwannoma, Castleman disease, retroperitoneal tumors.

\section{口 Introduction}

The retroperitoneal tumors are rare neoplasms that pose many difficulties for an accurate preoperative diagnosis. When located in pelvis, they can be misdiagnosed as uterine or adnexal masses. In gynecological practice, the most encountered tumor condition involving the uterus is represented by the uterine myoma. The clinical examination is, in many cases, inconclusive. Although the ultrasound (US) examination establishes the correct diagnosis in most of the cases, there are, in clinical practice, situations when other pelvic tumors can be preoperatively confounded with a uterine myoma. The errors in the diagnosis can lead to difficulties, especially during the surgical treatment, as retroperitoneal tumors need a complex surgical team, which includes both, gynecologist and general surgeon. The retroperitoneal tumors represent a challenge for the gynecologist not only because of their rarity, but also due to the anatomic complexity of the region [1]. A complete and detailed imagistic assessment of the pelvic structures is important in order to improve the outcome of the surgical treatment. However, because of overlapping of the imagistic aspects and lack of specific radiological features, these tumors can raise difficulties even to an experienced radiologist, and the final diagnosis is established at histopathological (HP) examination [2]. Usually, the solid benign tumors of retroperitoneum can mimic a uterine myoma due to similarities in ultrasonographic features, especially in cases of schwannomas and Castleman disease. The gynecologist should be aware of the possibility of a retroperitoneal tumor in order to take the correct decision regarding the referral of the patient.

The aim of our study is to present an up-date upon pathogenesis, pathology and management of these rare retroperitoneal tumors. Although rare, they need to be taken into consideration in the differential diagnosis of uterine myomas, especially in the pedunculated form.

\section{ㅁ Retroperitoneal tumors}

From the anatomical point of view, the retroperitoneum is defined as being a part of the abdominal cavity, anteriorly delimited by the parietal peritoneum and posteriorly by the transversalis fascia [3]. In the retroperitoneal space, different types of tumors can develop, some of them benign (lymphangioma, leiomyoma, benign pheochromocytoma, etc.), other malignant (lymphangiosarcoma, liposarcoma, leiomyosarcoma, malignant schwannoma, etc.) [2].

Retroperitoneal tumors usually arise from the tissues that form the retroperitoneal spaces rather the retroperitoneal organs. There are multiple classifications described

This is an open-access article distributed under the terms of a Creative Commons Attribution-NonCommercial-ShareAlike 4.0 International Public License, which permits unrestricted use, adaptation, distribution and reproduction in any medium, non-commercially, provided the new creations are licensed under identical terms as the original work and the original work is properly cited. 
in the literature, but from the clinical point of view, the most useful are those based on the tissue of origin and on the imagery data - solid or cystic. The mesodermal origin is encountered in the majority of cases; practically, most of the retroperitoneal tumors can arise from any tissue present in the retroperitoneum [3].

Generally, retroperitoneal tumors may appear at any age, but are more frequently in adults, with a maximum incidence at the age of $40[4,5]$. Studies have shown that malignant retroperitoneal tumors are more often encountered, being four times more frequent than benign tumors [6].

Among the most frequent retroperitoneal malignant tumors are the sarcomas, which occupy the first place [2]. In their retrospective study, Fujimoto et al. (2018) have analyzed the incidence of different types of retroperitoneal tumors, both benign and malignant, in a group of 167 patients. From the histological point of view, the results show that liposarcomas were the most frequent as incidence, followed by schwannomas, paragangliomas, and in fourth place, the leiomyosarcomas. Of all these, the most common benign tumors were the schwannomas [7]. Both, the low incidence of retroperitoneal tumors and the clinical picture, which is often poor, explain the difficulty the surgeon encounters when it comes to a positive or differential diagnosis [3].

\section{ㅁ Schwannomas}

Schwannomas or the so-called neuromas are known as benign tumors that arise from the Schwann cells of nerve fibers $[8,9]$. These tumors are rarely malignant $(<1 \%$ of cases) [10] and are frequently found in the head, neck and mediastinum, but sometimes also at the level of the extremities $[11,12]$. Studies have shown that the pelvic location of schwannomas is very rare $(3 \%)$ and in this case, they emerge from the sacral nerve or hypogastric plexus [12]. Sacral schwannomas can be classified in three categories: type I - when the tumor is confined to the sacrum; type II - when the tumor arise from the sacrum but involve also pre-sacral or subcutaneous space; type III - the tumor is located in the pelvis or retroperitoneum [13].

The Von Recklinghausen disease (neurofibromatosis type 1) is a dominantly autosomal hereditary disorder, which may influence the real incidence of schwannomas, located in retroperitoneum [14]; studies show that when these two pathologies coexist, the incidence of retroperitoneal schwannomas increases [15].

The etiology of schwannomas is not known yet [2], it affects people with ages between 20-50 years [8], more frequent in female patients [10]. Several tumor suppressor genes like SWI/SNF-related, matrix-associated, actindependent regulator of chromatin, subfamily $b$, member 1 (SMARCB1) and leucine zipper-like transcription regulator 1 (LZTR1) appear to be involved in the pathogenesis of the schwannomas, according to molecular studies of KehrerSawatzki et al. [16].

Generally, schwannomas localized in retroperitoneal space are asymptomatic. Because these tumors grow very slow [10], some are discovered accidentally [8]. Usually, schwannomas grows and extend along the length of the nerve where they originate [17]. Sometimes, because they are asymptomatic, they can reach very large size and even erode into adjacent bony structures [18].

When reaching a significant size, they can compress adjacent structures and therefore generate nonspecific signs and symptoms like abdominal pain and distension accompanied by lower back pain [19]. In other instances, digestive signs (nausea, vomiting and constipation) or venous and lymphatic obstruction may dominate the clinical picture [5]. The altered general condition, the fever and the weight loss can be signs of malignancy [5].

These non-specific signs and symptoms make the positive diagnosis a challenging one, which can mislead the surgeon and, sometimes, can lead to a misdiagnosis. Schwannomas are, e.g., sometimes confused with pelvic lesions [12].

An article recently published by Kalagi et al. (2019) exposes the case of a patient who was accidentally diagnosed with a schwannoma after a road accident; the retroperitoneal mass was found on the abdominal computed tomography (CT) exam and the diagnosis was subsequently confirmed by biopsy [19].

In some cases, the clinical picture may be more complex; for instance, in the article "Gynaecological perspective of schwannoma: a rare pelvic tumour", published in 2016, it is presented a case of a schwannoma in a 26-year-old female who complains of abdominal pain, dysmenorrhea with dyspareunia. At the physical examination, it was detected an abdominal mass with limited mobility pushing the cervix toward the pubic symphysis [8]. Usually, US examination is the first imagistic method used in clinical practice, although there are not specific sonographic signs for schwannomas. Usually, they are homogeneous, hypoechoic well-defined masses (Figure 1).

When the tumors are large $(>8 \mathrm{~cm})$ they can suffer cystic degeneration due to necrosis or hemorrhage. Doppler examination can detect blood flow in most of the cases [11] (Figure 2).

Huang et al. (2020) described the contrast-enhanced ultrasonography, in a case of schwannoma in the presacral space. With this technique, the tumor has a rich contrast intake, with a hyper-enhanced pattern compared to the surrounding tissue in arterial phase, the inner parts enhanced firstly and quickly the whole lesion enhanced except several non-enhanced areas [11].

CT scan can deliver more information in the diagnosis of schwannomas [20]. In 66\% of cases, CT scan can detect cysts and in some cases, calcifications can be observed [21]. The magnetic resonance imaging (MRI) findings can be helpful, but are non-specific. They are represented by an association of a $\mathrm{T} 1$ isosignal to the adjacent skeletal muscle, with a T2 hypersignal to the adjacent skeletal muscle. The main role of the MRI is to observe the limit between tumor and surrounding structures [11].

Although CT or MRI imaging examination plays an important role in finding the retroperitoneal tumors [3], but it should be mentioned that only the pathological examination could confirm the nature of the tumor [22].

Schwannomas are frequently confused with other types of retroperitoneal tumors, the differential diagnosis can be made with a liposarcoma, gastrointestinal stromal tumors $(<5 \%$ of cases) or non-Hodgkin's lymphoma [23, 24]. 
For instance, in the article "Schwannoma in pelvis: a diagnostic dilemma", published in 2017, Ray et al. (2017) present the case of a 40-year-old patient who complains of pain in the lower quadrant of the abdomen associated with dysuria. At the CT examination, a tumor mass was found in the pre-sacral region. Both the cystoscopy and the colonoscopy showed no pathological changes. A biopsy was performed and the results showed a positivity for S100 protein, which indicates that the tumor is of neuronal origin [23]. We specify the fact that the S100 protein is the best indicator used for the tumors originating from the peripheral nerve sheath [24] (Figure 3).

The gross appearance of schwannomas is represented by a well-delineated mass due to a peripheral collagenic capsule. Microscopic examination reveals degenerative areas intercalated with compact spindled and cystic areas. There are described Verocay bodies formed by palisades

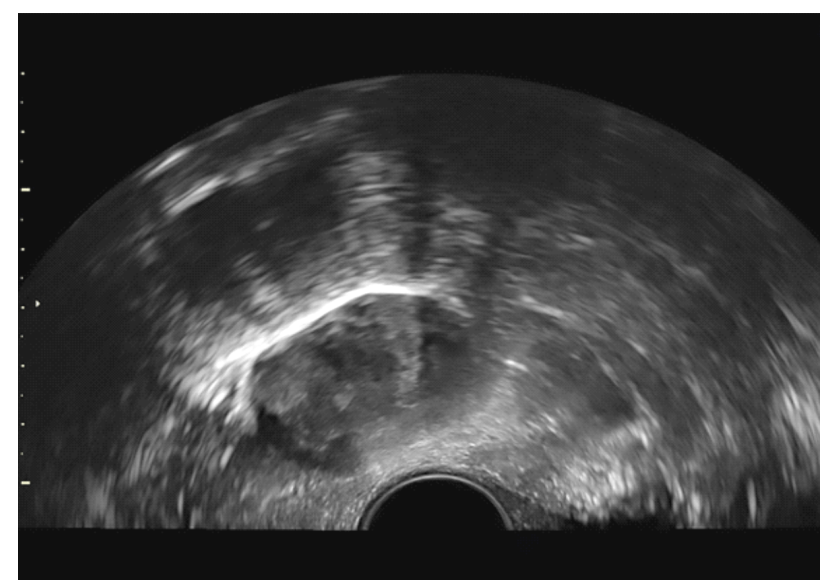

Figure 1 - Schwannoma, transvaginal ultrasound. A welldelineated solid mass can be seen in the pelvis, near the uterus.

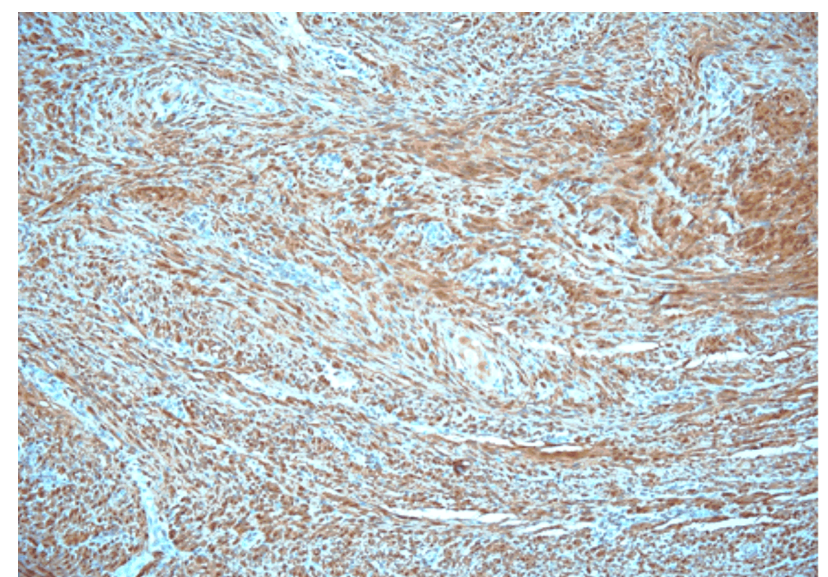

Figure 3 - Schwannoma. S100 positivity of the tumoral proliferation (Anti-S100 antibody immunostaining, $\times 200$ ).

A particular type is cellular schwannoma, characterized by high cellularity and increased mitotic activity. The main features are proliferation of well-differentiated Schwann cells and absence of Verocay bodies. Because of fascicular growth and locally destructive behavior, it can sometimes invade the bone structures. Another particular form is plexiform schwannoma a tumor, which is not well delineated, due to the lack of capsula. It has Antoni A pattern and a plexiform type growth. The diagnosis can of Schwann cell nuclei and a fibrillary core. Frequently are encountered hyalinized vessels [25].

Schwannomas can present several histological appearances and variants, which have been well described in the scientific literature [26]. In 1920, the Swedish neurologist Antoni histologically describes two different architectural patterns found in the peripheral nerve tumors. These two architectural patterns are suggestive for schwannomas and allow a positive diagnosis [27]. In the Antoni A type, the tissue is characterized by a compact hypercellular area and in the Antoni B type, the tissue is hypocellular with cystic changes. Antoni B type also bears the name of degenerate Antoni A [27].

The malignant transformation of the schwannoma is histologically characterized by: mitosis, pleomorphism and vascular infiltration [23] (Figure 4).

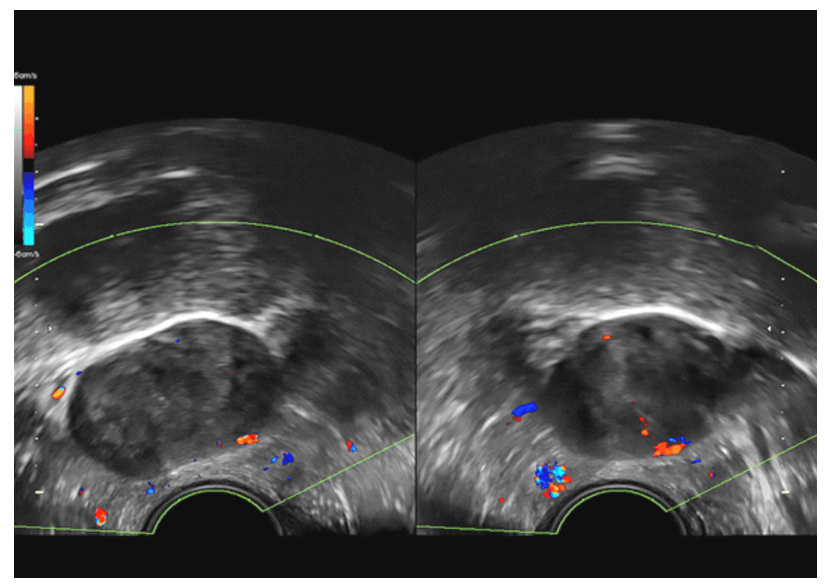

Figure 2 - Schwannoma. Doppler transvaginal ultrasound. Several vessels are visible at the periphery and in the interior of the mass.

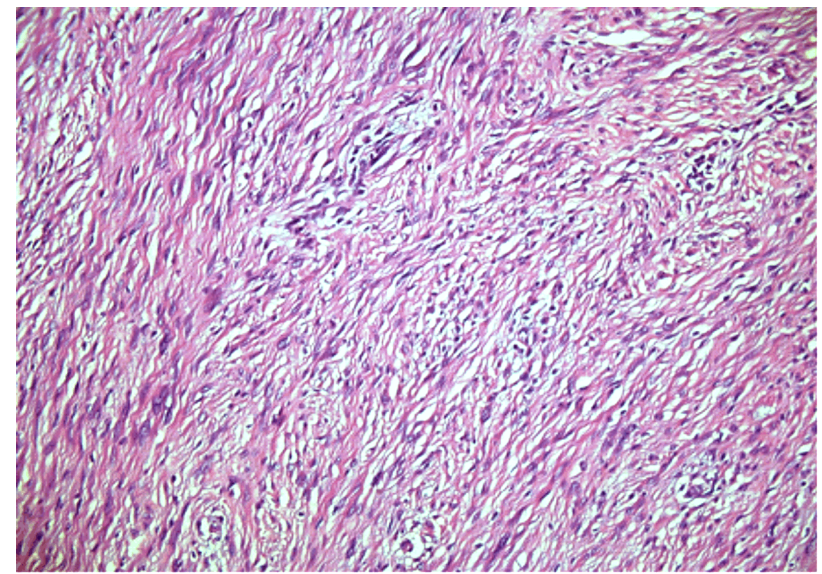

Figure 4 - Schwannoma. Spindle cell proliferation, with high cellularity and palisaded distribution [HematoxylinEosin (HE) staining, $\times 200]$.

be challenging in rare forms like reticular schwannoma, that has origin mainly in viscera and had myxoid pattern and microcysts [25].

Immunohistochemistry plays an important role in the diagnosis. Schwannomas are intensely stained with S100 protein, vimentin, and neuron-specific enolase, but not by smooth muscle actin (SMA) or cluster of differentiation (CD) 117 molecules [28].

Management of the schwannomas should always be 
represented by surgical removal, as early as possible in order to avoid excessive growth of the tumor and compressing the surrounding organs. The surgical removal should be ideally represented by total resection in order to improve the survival [29]. When the tumor is extended above the S3 level, in order to reduce the risk of damaging the sacral nerves a partial resection is recommended. For type III schwannomas, the anterior approach is recommended [29]. Laparoscopic approach can be used with similar outcomes as open surgery. In general, laparoscopic approach is recommended when the tumor is small $[30,31]$.

\section{ㅁ Castleman disease}

Castleman disease is a rare condition that affects the lymphatic system. It is characterized by an exaggerated hyperplasia of the lymph nodes, and is sometimes associated with herpes virus infection $[32,33]$. The etiopathology of the disease is not elucidated yet [32]. This condition was firstly described by Castleman \& Towne, in 1954, who reported a series of 13 subjects with localized enlargement of mediastinal hypervascular lymph nodes [34]. Castleman disease is rarely encountered in gynecology [32]. It is localized in the mediastinum in $70 \%$ of cases, but it can also be found in the neck, axilla, pelvis and, in a smaller percentage, in the retroperitoneum (7\%) [32].

From the clinical point of view, it is classified in: the localized form (90\%), the patients are usually asymptomatic, and the multifocal form $(10 \%)$. Each form is considered to represent a clinical entity, with separate risk factors, clinical symptoms and outcomes [35]. The localized or unicentric form is a benign condition, usually asymptomatic, characterized by a unique lymphoid mass, most often localized in the mediastinum and rarely in the neck or abdomen. The multifocal or multicentric form, which involves more than one lymphoid mass, is correlated, in many cases, with human herpes virus-8 (HHV-8) infection or with polyneuropathy, organomegaly, endo-

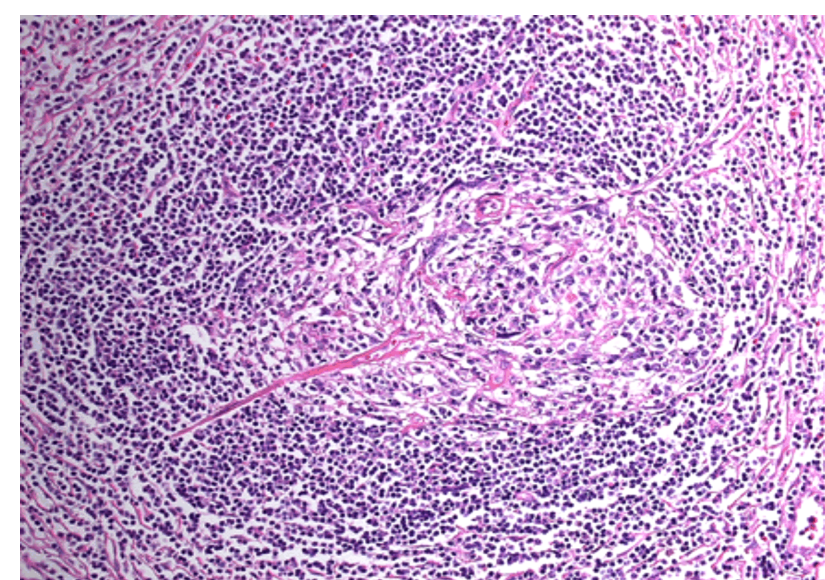

Figure 5 - Castleman disease. Lymphoid follicle with unpopulated germinal center and penetrating hyaline artery (HE staining, $\times 200)$.

In hypervascular pattern, which characterizes multicentric disease, the follicles have an abnormal aspect with both atretic and sclerotic changes. There is a disproportion between B-cells, which are in a few number and dendritic cells. The B-cell can also be displayed concentrically around the follicle giving the same "onion skin" appearance as crinopathy, monoclonal gammopathy, and skin changes (POEMS) syndrome [32, 36]. However, one-third of multicentric form cases are human immunodeficiency virus (HIV) and HHV-8 negative, which is known as idiopathic multicentric Castleman disease [37].

The histological pattern may differ according the clinical classification. There are described three HP forms: hyaline-vascular, which is usually encountered in unicentric disease, hypervascular and the plasma cell form, which are characteristic for multicentric forms. Sometimes, these three patterns can be present altogether. In unicentric disease, usually only one lymph node is affected and it can increase up to size of $6-8 \mathrm{~cm}$ and in more than $90 \%$ of the cases the histological pattern superpose on the hyaline-vascular pattern, while $10 \%$ of the cases have marked plasmacytosis, which is specific for multicentric form of the disease [38].

In hyaline-vascular pattern, the follicles are small due to a depletion of lymphoid cells, centered by the hyalinized arteries with capillary proliferation. An increase in follicles density and abnormalities in the architectural pattern might be noticed. Follicular dendritic cells that may show cytological atypia might be a marker of this variant. The follicles are surrounded by lymphocytes with a concentrically display, which give the appearance of an "onion skin"-like picture. If the concentric mantle formed by lymphocytes includes two atretic follicles, the "twinning" feature is described (Figures 5 and 6). Sometimes, a "lollipop" pattern is described due to penetrating blood vessels with a radially arrangement in the follicle. A marked proliferation of the vascular network characterizes the hyaline-vascular type due to both an increase density of the vessels and enlargement of the endothelial cells. The lymph node sinuses are absent and this is an important HP feature, useful to make the difference between hyaline-vascular and hypervascular variant [38].

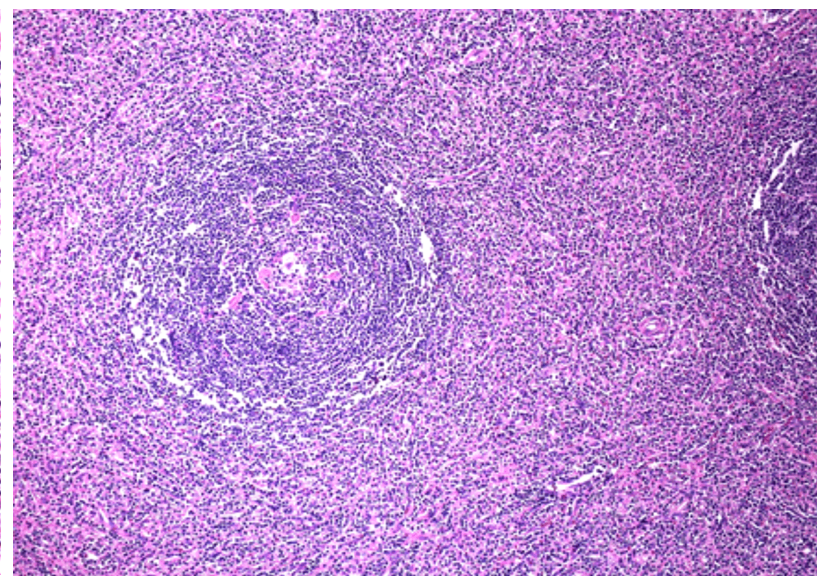

Figure 6 - Castleman disease. Section through the mantle zones of a lymphoid follicle with an "onion-skin" appearance (HE staining, $\times 200)$.

in hyaline-vascular pattern. A marked vascularization is present due to an increased number of high endothelial venules. The presence of lymph node sinuses makes the difference between the hypervascular and hyaline-vascular form [38].

In plasma cell form, a specific feature is an increase 
in mature plasma cells between the follicles present as large aggregates or confluent sheets. The normal pattern of the lymph node is maintained and a reactive follicular hyperplasia is observed. There is less vascular proliferation in comparison with hypervascular pattern [38].

Recently, two subtypes were described: HHV-8 associated type (especially in HIV-patients), and the multicentric not otherwise specified (NOS) type [39, 40]. In the HHV-8 type, the lymph node architecture is generally normal and either follicle hyalinization or marked interfollicular vasculature can be noticed. The infected plasma cells surround the nodal follicles and are characterized by amphophilic cytoplasm and normal or increased size [38].

Studies have shown that the incidence of the disease is almost 15 per one million patients/year [41], but it should be taken into consideration when it comes to a differential diagnosis in a young patient who at imaging exams presents a retroperitoneal mass.

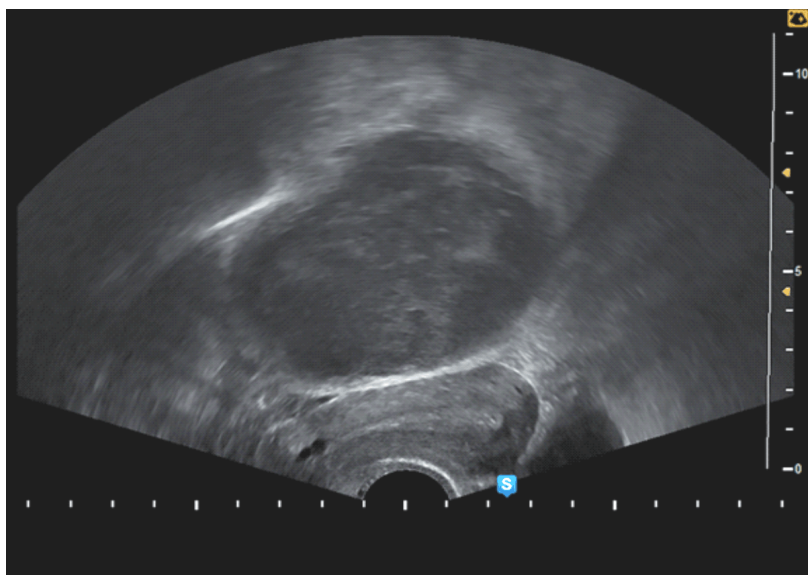

Figure 7 - Castleman disease. Transvaginal ultrasound. Longitudinal plane of the uterus. A well-delineated solid mass in contact with the posterior wall of the uterus.

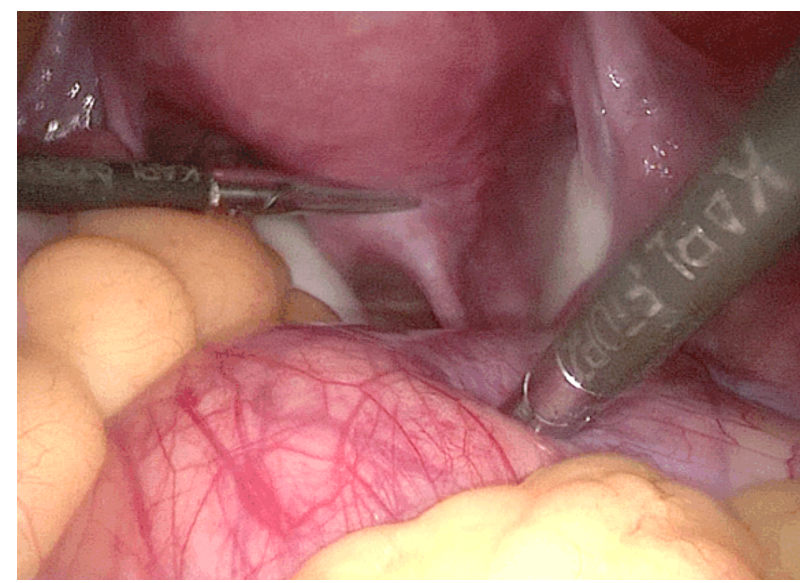

Figure 9 - Castleman disease. Laparoscopy reveals a large retroperitoneal mass in the hypogastric area, and a normal uterus.

The CT shows the same well-circumscribed image, with homogeneous soft-tissue attenuation, marked or moderated enhancement in the post-contrast arterial phase and punctate calcifications. MRI examination includes T1-hypointensity, T2-hyperintensity and contrast enhancement after gadolinium administration. Because these
The clinical picture of a patient with Castleman disease is often non-specific. The unicentric form is usually asymptomatic, while the multicentric form is characterized by increased serum interleukin-6 (IL-6) levels, and a several systemic manifestations [42]. Multicentric disease, which usually affects adult ages of 50-60 years, is characterized by a systemic symptomatology: fever, weakness, weight loss and peripheral lymphadenopathy [43]. The prognosis of unicentric disease is better than in multicentric disease, which is more aggressive and less likely to be cured by surgical resection [34].

Sometimes, the pain may be the only symptom. Imaging (CT, MRI) may indicate the shape, size, and location of the lesion, but does not allow establishing a positive diagnosis of Castleman disease [32].

Imaging features of unicentric disease are not specific. US examination shows a unique, well-defined mass, sometimes with hypechogenic foci due to calcifications (Figures 7-9).

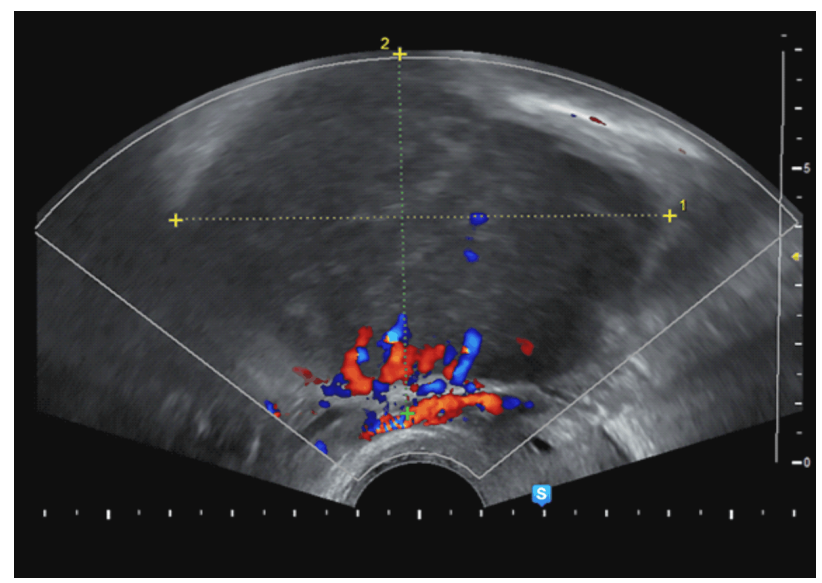

Figure 8 - Castleman disease. Transvaginal ultrasound. Longitudinal plane of the uterus. Several vessels are visible between the uterus and the mass, giving the impression that the mass is originating from the uterus.

images are not specific, several other diseases should be taken into consideration, like: lymphomas, retroperitoneal sarcoma, desmoid tumors and metastatic disease [44]. The differential diagnosis in multicentric disease includes: lymphomas, infections and immunological diseases like systemic lupus erythematosus. The persistent generalized lymphadenopathies in HIV-seropositive subjects could be misdiagnosed as Castelman, multicentric disease [45]. The positron emission tomography (PET)-CT shows an increase in glucose intake in lymphoid masses, however the uptake has a low level in comparison with lymphomas [45].

The positive diagnosis is challenging, and is especially based on the histological examination. Various published articles attest the fact that a pre-operative diagnosis is difficult and it requires special attention.

Karaman et al. published in 2018 a case of a 52-yearold patient presenting abdominal pain in the lower left quadrant, without other associated signs and symptoms. The abdominal US showed an adnexal solid mass similar to a uterine leiomyoma. Subsequently, the suspicion of a fibrothecoma originating from the left ovary was excluded after the MRI scan. The intraoperatory findings were as follows: enlarged uterus with adenomyosis, ovaries with normal aspect and the peritoneum was covering a solid 
mass at the level of the left iliac artery. In this case, we can state that the MRI examination could not differentiate between a Castleman disease and an adnexal tumor. The biopsy performed has allowed a positive diagnosis of plasma cell Castleman disease. It is not a rule, the presented case is an exception, but in many cases, a patient with Castleman disease located in the pelvic region has no symptoms and, frequently, the disease has a hyalinevascular form [46].

Nakata et al. (2020) published a case of a 47-yearold patient, totally asymptomatic, who presents a tumor mass accidentally found on a X-ray examination. The transvaginal US examination revealed a left ovarian cyst and at the MRI examination, a homogeneous left retroperitoneal tumor of $5 \mathrm{~cm}$ was found [33]. Intraoperatory, the patient presented an external iliac lymphadenopathy, and the tumor previous described had a firm yellow consistency. The histological examination described a follicular hyperplasia with marked hyalinization and an expanded follicular dendritic cell network. The immunofluorescence exam revealed a high positivity for CD20 B-lymphocytes and CD3 T-lymphocytes and negativity for immunoglobulin G4 (IgG4). Therefore, the diagnosis of unifocal Castleman disease with hyaline-vascular form was confirmed. As a particularity of the case, we have to mention that the identification of the origin of the retroperitoneal mass was facilitated by the fact that the patient had an ipsilateral ovarian cyst [33].

Abdessayed et al. (2017) published another case of a 34-year-old patient complaining of epigastric pain, especially postprandial. The results of the biochemistry examinations did not reveal anything significant. A tumor, which seemed to be localized in the pancreas, was found by the ultrasonographic examination. At the CT examination, this tumor had a homogeneous appearance; it was solid and well delineated, adjacently located to the celiac trunk. This time there was the suspicion of either a tumor at the level of the pancreas or a gastrointestinal stromal tumor. As in the above-mentioned cases, the diagnosis was reoriented to another form of the disease, the pancreas, stomach, and liver being normal at the intraoperative inspection. The HP examination showed large lymphoid follicles in the form of "onion skin", atrophic germinal centers with concentric rings of B-lymphocytes. On the other hand, a hyalinization at the stromal level was found, without an aggregation of the plasma cells [47].

All the cases taken from literature and presented in the current paper have not shown general signs and symptoms (fever, anemia, thrombocytopenia or weight loss), and the pain was a dominant symptom. No imaging method could state a positive diagnosis; the HP examination was always necessary.

The surgical management of the unicentric disease should be represented by a complete removal of the mass, in order to achieve nearly a $100 \% 5$-year survival. After surgical removal, the clinical symptoms will disappear and the seric level of IL-6 decreases [48]. If the complete removal cannot be achieved, the alternative is represented by radiation therapy [40]. It is difficult to treat and completely eradicate the multicentric disease. In certain cases, corticosteroid, chemotherapy, monoclonal antibodies and radiotherapy can be applied [42].
After surgery, the patients should be closely monitored by clinical examination, imagistic exams and serum markers, due to the risk of lymphomatous conversion of the lymphoid masses or the development of lymphomas in other sites. It is recommended an annual ${ }^{18} \mathrm{~F}$-labeled fluoro-2-deoxyglucose $\left({ }^{18} \mathrm{~F}-\mathrm{FDG}\right)-\mathrm{PET}$ and serum biomarkers (IL-6, $\mathrm{C}$-reactive protein, serum free light chains, and quantitative immunoglobulins) [49].

\section{ㅁ Conclusions}

The imaging methods are not always conclusive for the final positive diagnosis. The CT examination may be helpful in finding suggestive changes for retroperitoneal schwannomas like cystic degeneration, calcifications or necrosis areas. Other characteristic, but non-specific changes at MRI examination might be the tumor mass identified in the T1 hypodense and T2 hyperdense signal. Taking into consideration, the fact that schwannomas and Castleman disease have no specific symptomatology, it is advisable to include these conditions in the differential diagnosis of the uterine myomas, especially in the pedunculated type.

\section{Conflict of interests}

The authors declare that they have no conflict of interests.

\section{References}

[1] Wee-Stekly WW, Mueller MD. Retroperitoneal tumors in the pelvis: a diagnostic challenge in gynecology. Front Surg, 2014, 1:49. https://doi.org/10.3389/fsurg.2014.00049 PMID: 25593973 PMCID: PMC4286971

[2] Scali EP, Chandler TM, Heffernan EJ, Coyle J, Harris AC, Chang SD. Primary retroperitoneal masses: what is the differential diagnosis? Abdom Imaging, 2015, 40(6):1887-1903. https://doi.org/10.1007/s00261-014-0311-x PMID: 25468494

[3] Selçuk I, Ersak B, Tatar İ, Güngör T, Huri E. Basic clinical retroperitoneal anatomy for pelvic surgeons. Turk J Obstet Gynecol, 2018, 15(4):259-269. https://doi.org/10.4274/tjod. 88614 PMID: 30693143 PMCID: PMC6334244

[4] Mota MMDS, Bezerra ROF, Garcia MRT. Practical approach to primary retroperitoneal masses in adults. Radiol Bras, 2018, 51(6):391-400. https://doi.org/10.1590/0100-3984.2017.0179 PMID: 30559557 PMCID: PMC6290739

[5] Aitsakel A, Elsayegh H, Benslimane L, Nouini Y. Tumeurs rétropéritonéales primitives : profils diagnostique, thérapeutique et anatomopathologique. Can Urol Assoc J, 2015, 9(11-12): E873-E878. https://doi.org/10.5489/cuaj.2161 PMID: 26788238 PMCID: PMC4709243

[6] Strauss DC, Hayes AJ, Thomas JM. Retroperitoneal tumours: review of management. Ann $\mathrm{R}$ Coll Surg Engl, 2011, 93(4): 275-280. https://doi.org/10.1308/003588411X571944 PMID: 21944791 PMCID: PMC3363075

[7] Fujimoto N, Kubo T, Hisaoka M, Udo K, Yokomizo A, Shibuya T, Wakeda H, Nishihara K, Moriya R, Iwakuma K; Kyushu Cooperative Urological Research Group. Demographics, management and treatment outcomes of benign and malignant retroperitoneal tumors in Japan. Int J Urol, 2018, 25(1):61-67. https://doi.org/10.1111/iju.13469 PMID: 28994196

[8] Padmanaban N, Chandrabose PS, Esakki M, Kirubamani H, Srinivasan C. Gynaecological perspective of schwannoma: a rare pelvic tumour. J Clin Diagn Res, 2016, 10(4):QD03QD05. https://doi.org/10.7860/JCDR/2016/16301.7587 PMID: 27190900 PMCID: PMC4866198

[9] Murărescu ED, Ivan L, Mihailovici MS. Neurofibroma, schwannoma or a hybrid tumor of the peripheral nerve sheath? Rom J Morphol Embryol, 2005, 46(2):113-116. PMID: 16286996

[10] Caballero Martel JR, Estévez Sarmiento S. Schwannoma: a rare Hoffa's fat pad tumor. Surg J (N Y), 2019, 5(2):e62-e64. https://doi.org/10.1055/s-0039-1692996 PMID: 31367680 PMCID: PMC6663472 
[11] Huang M, Qian H, Wang J, Zhao Q, Jiang T. Giant presacra schwannoma in man: report of a case with emphasis on imaging findings. World Neurosurg, 2020, 133:14-16. https:// doi.org/10.1016/j.wneu.2019.09.074 PMID: 31557553

[12] Pak H, Haji Maghsoudi L. Pelvic schwannoma: in light of a case report. Clin Case Rep, 2019, 7(12):2488-2490. https:// doi.org/10.1002/ccr3.2542 PMID: 31893085 PMCID: PMC 6935657

[13] Klimo P Jr, Rao G, Schmidt RH, Schmidt MH. Nerve sheath tumors involving the sacrum. Case report and classification scheme. Neurosurg Focus, 2003, 15(2):E12. https://doi.org/ 10.3171/foc.2003.15.2.12 PMID: 15350043

[14] Adil A, Singh AK. Neurofibromatosis type 1 (Von Recklinghausen). In: ${ }^{* *}$. StatPearls [Internet]. StatPearls Publishing, Treasure Island, FL, USA, Jan 2020, updated: May 2020, available from: https://www.ncbi.nlm.nih.gov/books/NBK459 358/. PMID: 29083628

[15] Machairiotis N, Zarogoulidis P, Stylianaki A, Karatrasoglou E, Sotiropoulou G, Floreskou A, Chatzi E, Karamani A, Liapi G, Papakonstantinou E, Katsikogiannis N, Courcoutsakis N, Machairiotis C. Pelvic schwannoma in the right parametrium. Int J Gen Med, 2013, 6:123-126. https://doi.org/10.2147/ IJGM.S41224 PMID: 23515244 PMCID: PMC3598498

[16] Kehrer-Sawatzki H, Farschtschi S, Mautner VF, Cooper DN. The molecular pathogenesis of schwannomatosis, a paradigm for the co-involvement of multiple tumour suppressor genes in tumorigenesis. Hum Genet, 2017, 136(2):129-148. https:// doi.org/10.1007/s00439-016-1753-8 PMID: 27921248 PMCID: PMC5258795

[17] Budu VA, Bulescu IA, Popp CG, Mocanu BC, Mogoantă CA. Vagus nerve schwannoma in the parapharyngeal space: surgical, histological and immunohistochemical aspects. A case report. Rom J Morphol Embryol, 2015, 56(1):273-276. PMID: 25826516

[18] Getachew MM, Whitman GJ, Chew FS. Retroperitoneal schwannoma. AJR Am J Roentgenol, 1994, 163(6):1356. https:// doi.org/10.2214/ajr.163.6.7992727 PMID: 7992727

[19] Kalagi D, Bakir M, Alfarra M, Aborayya A, Anwar I. Two unusual presentations of presacral schwannoma; a case series. Int J Surg Case Rep, 2019, 61:165-168. https://doi. org/10.1016/j.ijscr.2019.07.042 PMID: 31374466 PMCID: PMC6675969

[20] Meşină C, Mogoantă SŞ, Cristian DA, Dumitrescu TV, Drăgoescu PO, Meşină-Botoran MI, Ciurea ME, Ghiluşi MC, Ciobanu D. Retroperitoneal ancient schwannoma - case presentation. Rom J Morphol Embryol, 2015, 56(4):15171522. PMID: 26743303

[21] Kinoshita T, Naganuma H, Ishii K, Itoh H. CT features of retroperitoneal neurilemmoma. Eur J Radiol, 1998, 27(1):67-71. https://doi.org/10.1016/s0720-048x(97)00032-6 PMID: 9587770

[22] Brennan C, Kajal D, Khalili K, Ghai S. Solid malignant retroperitoneal masses - a pictorial review. Insights Imaging 2014, 5(1):53-65. https://doi.org/10.1007/s13244-013-0294-0 PMID: 24293303 PMCID: PMC3948907

[23] Ray M, Jayakumar P, Mishra A, Gowda M, Padmanabhan N. Schwannoma in pelvis: a diagnostic dilemma. Indian J Gynecol Oncolog, 2017, 15(3):46. https://doi.org/10.1007/s40944-0170133-1

[24] Kameyama H, Kanda T, Tajima $Y$, Shimada $Y$, Ichikawa $H$, Hanyu T, Ishikawa T, Wakai T. Management of rectal gastrointestinal stromal tumor. Transl Gastroenterol Hepatol, 2018 3(2):8. https://doi.org/10.21037/tgh.2018.01.08 PMID: 29552659 PMCID: PMC5847932

[25] Rodriguez FJ, Folpe AL, Giannini C, Perry A. Pathology of peripheral nerve sheath tumors: diagnostic overview and update on selected diagnostic problems. Acta Neuropathol, 2012, 123(3):295-319. https://doi.org/10.1007/s00401-0120954-z PMID: 22327363 PMCID: PMC3629555

[26] Georgescu TA, Dumitru AV, Oproiu AM, Nica AE, Costache D, Pătraşcu OM, Lăzăroiu AM, Chefani AE, Sajin M, Costache $M$ Cutaneous microcystic/reticular schwannoma: case report and literature review of an exceedingly rare entity with an unusual presentation. Rom J Morphol Embryol, 2018, 59(1): 303-309. PMID: 29940642

[27] Wippold FJ 2nd, Lubner M, Perrin RJ, Lämmle M, Perry A. Neuropathology for the neuroradiologist: Antoni $A$ and Antoni $B$ tissue patterns. AJNR Am J Neuroradiol, 2007, 28(9):16331638. https://doi.org/10.3174/ajnr.A0682 PMID: 17893219
[28] Strauss DC, Qureshi YA, Hayes AJ, Thomas JM. Management of benign retroperitoneal schwannomas: a single-center experience. Am J Surg, 2011, 202(2):194-198. https://doi.org/ 10.1016/j.amjsurg.2010.06.036 PMID: 21810500

[29] Pennington Z, Westbroek EM, Ahmed AK, Cottrill E, Lubelski D, Goodwin ML, Sciubba DM. Surgical management of giant presacral schwannoma: systematic review of published cases and meta-analysis. J Neurosurg Spine, 2019, 31(5):711-722. https://doi.org/10.3171/2019.4.SPINE19240 PMID: 31277062

[30] Di Furia M, Salvatorelli A, Della Penna A, Vicentini V, Sista F, Chiominto A, Guadagni S, Clementi M. Advantage of laparoscopic resection for pelvic schwannoma: case report and review of the literature. Int J Surg Case Rep, 2018, 45:38-41. https://doi.org/10.1016/j.ijscr.2018.03.006 PMID: 29571063 PMCID: PMC6000728

[31] Chopra S, Dharmaraja A, Satkunasivam R, Gill IS. Robotassisted laparoscopic resection of a pelvic schwannoma. Urol Case Rep, 2017, 11:63-65. https://doi.org/10.1016/j.eucr. 2016.11.027 PMID: 28180092 PMCID: PMC5293720

[32] Karalök A, Taşçı T, Üreyen I, Koç S, Boran N, Çavuşoğlu D, Turan T, Tulunay G. Castleman disease mimicking ovarian tumour. Gynecol Obstet Reprod Med, 2016, 22(2):105-107. https://doi.org/10.21613/GORM.2016.481

[33] Nakata K, Iwahashi N, Matsukawa H, Noguchi T, Yahata T, Ota N, Mabuchi Y, Ino K. Laparoscopically resected Castleman's disease in the pelvic retroperitoneum: a case report. Mol Clin Oncol, 2020, 12(2):169-173. https://doi.org/10.3892/mco.20 19.1963 PMID: 31929889 PMCID: PMC6951224

[34] Schelble AP, Merritt DF. Pelvic Castleman's disease presenting as an adnexal mass in an adolescent. J Pediatr Adolesc Gynecol, 2019, 32(1):86-89. https://doi.org/10.1016/j.jpag. 2018.09.002 PMID: 30223024

[35] Talat N, Belgaumkar AP, Schulte KM. Surgery in Castleman's disease: a systematic review of 404 published cases. Ann Surg, 2012, 255(4):677-684. https://doi.org/10.1097/SLA.0b 013e318249dcdc PMID: 22367441

[36] Wojtyś M, Piekarska A, Kunc M, Ptaszyński K, Biernat W, Zaucha JM, Waloszczyk P, Lisowski P, Kubisa B, Grodzki T. Clinicopathological comparison and therapeutic approach to Castleman disease - a case-based review. J Thorac Dis, 2019, 11(11):4859-4874. https://doi.org/10.21037/jtd.2019.10. 73 PMID: 31903277 PMCID: PMC6940266

[37] Liu AY, Nabel CS, Finkelman BS, Ruth JR, Kurzrock R, van Rhee F, Krymskaya VP, Kelleher D, Rubenstein AH, Fajgenbaum DC. Idiopathic multicentric Castleman's disease: a systematic literature review. Lancet Haematol, 2016, 3(4):e163e175. https://doi.org/10.1016/S2352-3026(16)00006-5 PMID: 27063975

[38] Wu D, Lim MS, Jaffe ES. Pathology of Castleman disease. Hematol Oncol Clin North Am, 2018, 32(1):37-52. https:// doi.org/10.1016/j.hoc.2017.09.004 PMID: 29157618

[39] Polizzotto MN, Uldrick TS, Wang V, Aleman K, Wyvill KM, Marshall V, Pittaluga S, O'Mahony D, Whitby D, Tosato G, Steinberg SM, Little RF, Yarchoan R. Human and viral interleukin- 6 and other cytokines in Kaposi sarcoma herpesvirusassociated multicentric Castleman disease. Blood, 2013, 122(26):4189-4198. https://doi.org/10.1182/blood-2013-08519959 PMID: 24174627 PMCID: PMC3868925

[40] Guthrie PJ, Thomas JV, Peker D, Turkbey B, Rais-Bahrami S. Perivesical unicentric Castleman disease initially suspected to be metastatic prostate cancer. Urol Ann, 2016, 8(2):245-248. https://doi.org/10.4103/0974-7796.177196 PMID: 27141204 PMCID: PMC4839251

[41] Simpson D. Epidemiology of Castleman disease. Hematol Oncol Clin North Am, 2018, 32(1):1-10. https://doi.org/10. 1016/j.hoc.2017.09.001 PMID: 29157611

[42] Soumerai JD, Sohani AR, Abramson JS. Diagnosis and management of Castleman disease. Cancer Control, 2014 , 21(4):266-278. https://doi.org/10.1177/107327481402100403 PMID: 25310208

[43] Roca B. Castleman's disease. A review. AIDS Rev, 2009, 11(1):3-7. PMID: 19290029

[44] Smedile A, Capuano F, Fraticelli S, Lucioni M, La Fianza A. Unicentric or multicentric Castleman disease? A case report of a pelvic intraperitoneal mass in a middle aged woman. J Radiol Case Rep, 2019, 13(3):28-36. https://doi.org/10. 3941/jrcr.v13i3.3387 PMID: 31565175 PMCID: PMC6743853 
[45] Bonekamp D, Horton KM, Hruban RH, Fishman EK. Castleman disease: the great mimic. RadioGraphics, 2011, 31(6):17931807. https://doi.org/10.1148/rg.316115502 PMID: 21997995

[46] Karaman E, Ateş Ç, Kolusarı A, Alkış I, Şahin HG, Gül A, Demir F. A rare case of pelvic Castleman's disease mimicking an adnexal tumor. East J Med, 2018, 23(4):344-346. https:// doi.org/10.5505/ejm.2018.61580

[47] Abdessayed N, Bdioui A, Ammar H, Gupta R, Mhamdi N, Guerfela M, Mokni M. Retroperitoneal unicentric Castleman's disease: a case report. Int J Surg Case Rep, 2017, 31:54-57. https://doi.org/10.1016/j.jijscr.2016.12.023 PMID: 28107758 PMCID: PMC5247283
[48] Yoshizaki K, Murayama S, Ito H, Koga T. The role of interleukin-6 in Castleman disease. Hematol Oncol Clin North Am, 2018, 32(1):23-36. https://doi.org/10.1016/j.hoc. 2017.09.003 PMID: 29157617

[49] Madan R, Chen JH, Trotman-Dickenson B, Jacobson F, Hunsaker A. The spectrum of Castleman's disease: mimics, radiologic pathologic correlation and role of imaging in patient management. Eur J Radiol, 2012, 81(1):123-131. https://doi. org/10.1016/j.ejrad.2010.06.018 PMID: 20643523

\section{Corresponding authors}

Claudia Florida Costea, Associate Professor, MD, PhD, Department of Ophthalmology, Faculty of Medicine, Grigore T. Popa University of Medicine and Pharmacy, 16 University Street, 700115 laşi, Romania; Phone +40744-972 648, e-mail: costea10@yahoo.com

Alexandru Cărăuleanu, Senior Lecturer, MD, PhD, Department of Obstetrics and Gynecology, Faculty of Medicine, Grigore T. Popa University of Medicine and Pharmacy, 16 University Street, 700115 laşi, Romania; Phone +40744-517 106, e-mail: acarauleanu@yahoo.com 